\title{
The mechanism of intramolecular energy transfer in terminally substituted polyene molecules
}

\author{
N. Holl, H. Port, H.C. Wolf * \\ 3. Physikalisches Institut der Universität Stuttgart, Pfaffenwaldring 57, D-70550 Stuttgart, Germany \\ H. Strobel and F. Effenberger * \\ Instıtut für Organische Chemie der Universität Stuttgart, Pfaffenwaldring 55, D-70550 Stuttgart, Germany
}

Received 18 March 1993; in final form 15 June 1993

\begin{abstract}
Absorption, fluorescence and excitation spectra of different terminally substituted polyenes have been recorded in the UV-Vis region. The observed intramolecular unidirectional energy transfer from a donor substituent to an acceptor at the other end of the chain is influenced but not inhibited by incorporation of a spacer into the polyene chain. In molecules without a spacer, internal conversion within the supermolecule can explain the observed transfer of energy. In molecules with a spacer interrupting the polyene chain the observed intramolecular energy transfer can be explained in terms of Förster transfer.
\end{abstract}

\section{Introduction}

Unidirectional transfer of excitation energy within a molecule or a molecular functional unit (supermolecule) is a problem of high actual interest. Such processes are important for many biological systems. The primary step of photosynthesis is the most prominent example. There is also the idea that information transfer and storage on the molecular level might be possible in the context of using molecular systems as elements of molecular electronics. For a collection of references we refer to more recent review papers [13].

Our investigations in this field are mainly concerned with the synthesis and spectroscopic investigation of terminally donor-acceptor substituted polyene molecules with various chain length. The basic idea is relatively simple. The two end groups and the polyene chain in the center of the supermolecule can be considered as more or less independent units. If the lowest excited state of the acceptor group can act as trap for the energy of excitation of the donor group, these compounds should be appropriate

\footnotetext{
* Corresponding author.
}

models for an intramolecular transfer between donor and acceptor via the polyene chain.

In earlier papers we have been able to show that an energy transfer in supermolecules can be identified $[4,5]$. Measurements of quantum yields of acceptor fluorescence, in molecules in which a bicyclo [2.2.2] octane unit incorporated as spacer into the polyene chain in order to suppress energy conduction through the chain, are also reported [6]. The results can be summarized as follows (see also ref. [1]): In terminally substituted polyene molecules (up to 9 double bonds) with an anthryl (A) group as donor and tetraphenylporphyrine (TPP) as acceptor, intramolecular energy transfer has been identified. Selective excitation of the donor is possible and the transfer is not inhibited by the spacer.

There remain a number of unresolved problems. First, the total quantum yield of fluorescence emission is low due to unidentified radiationless processes. This makes an unambiguous quantitative analysis of energy flow within the supermolecules difficult. A second difficulty arises from the fact that selective excitation of the donor is not perfect. There is always a contribution from direct excitation of the acceptor. Furthermore, residual amounts of impurities cannot be excluded completely. If these exhibit 
relatively high fluorescence quantum yields, a quantitative fluorescence analysis is made extremely difficult [7]. Therefore, fluorescence decay measurements are also less conclusive.

\section{Outline}

The present paper is a report on additional experiments which are designed to address these problems.

First, increased effort was paid to sample purity and the presence of isomers. Second, experiments were performed in order to separate the energy transfer process from competitive radiationless relaxation. Finally, we varied both the position of the spacer (choosing the steroid androstane) within the polyene chain and the polyene chain length.

\section{Experimental}

\subsection{Molecules}

This paper concentrates on the supermolecules shown in fig. 1 . In addition to the compounds 1 to 7 similar molecules with different polyene chain lengths were investigated [6]. The conjugated supermolecules 1-3 and 7 consist of a single polyene chain in an all-trans configuration, stabilized by methyl side groups and with different terminal groups [8]. In addition, compounds 4-6 contain the spacer molecule androstane. In molecule 4, the androstane spacer is incorporated into the conjugated system. However, in the supermolecules 5 and $\mathbf{6}$, the androstane unit is directly bonded at one side to anthracene and with the other end to the polyene chain leading to a steric inversion of the steroid spacer in comparison to 4 . Contrary to the other compounds, the polyene chain in 6 is not stabilized by methyl side groups.

\subsection{Synthesis}

The polyenes 1-3 were synthesized via Wittig olefinations $[8,9]$ and were purified either by chromatography (HPLC and MPLC) $(1,3)$ or by combined chromatography and recrystallization (2) [8]. A de-
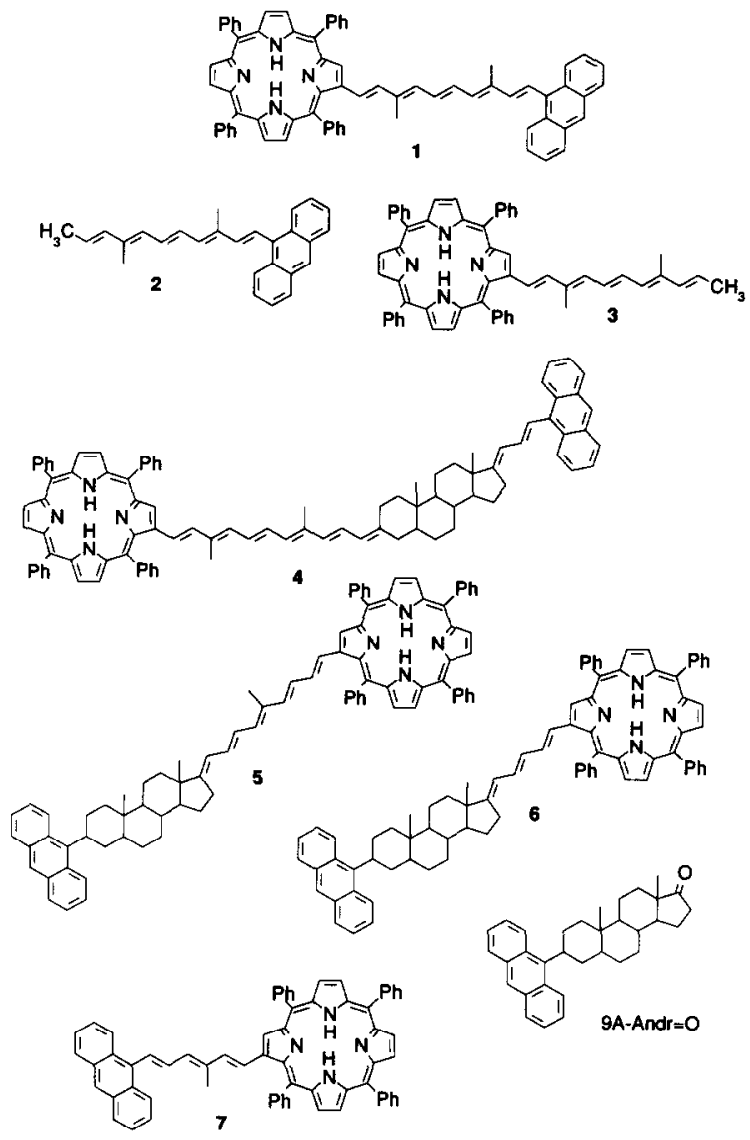

Fig. 1. Molecular structures of the supermolecules $1\left(9 \mathrm{~A}-\mathrm{P}_{5}-\right.$ TPP), $2\left(9 \mathrm{~A}-\mathrm{P}_{5}-\mathrm{CH}_{3}\right), 3\left(\mathrm{TPP}-\mathrm{P}_{5}-\mathrm{CH}_{3}\right), 4\left(9 \mathrm{~A}-\mathrm{P}_{2}-\mathrm{Andr}-\mathrm{P}_{6}-\right.$ TPP), 5 (9A-Andr-P - TPP), 6 (9A-Andr-P $\mathrm{P}_{3}-\mathrm{TPP}$ ), 7 (9A$P_{3}-$ TPP) and of the compound $9 \mathrm{~A}-\mathrm{Andr}=0$.

tailed publication [10] contains all aspects concerning the synthesis of compounds 4-7.

\subsection{Methods}

As solvent, $n$-hexane (Merck UVASOL) was used as purchased. The samples were prepared as liquid solutions with a concentration of $10^{-7}$ to $10^{-4}$ $\mathrm{mol} / \mathrm{\ell}$ in order to maintain low optical densities. The solutions were degassed from oxygene by repeated freeze-pump-thaw cycles and treated under inert gas atmosphere in the dark. Absorption spectra were recorded at $T=295 \mathrm{~K}$ using a Perkin Elmer Lambda 16 UV-Vis spectrometer. Emission $(T=180 \mathrm{~K})$ and 
excitation ( $T=295 \mathrm{~K}$ ) spectra were obtained with a $450 \mathrm{~W}$ xenon lamp and a $0.25 \mathrm{~m}$ double monochromator as light source, and a $1 \mathrm{~m}$ double monochromator with cooled photomultiplier as detection set-up. The photomultiplier was connected to a photon counter. All spectra presented in this work are corrected for the spectral responses of the equipment and represent quantum fluxes [11]. Fluorescence quantum yields were measured relative to a common standard, anthracene in $n$-hexane $\left(\eta_{\mathrm{f}}=0.30\right.$ at $T=295 \mathrm{~K}$ [12], $\eta_{\mathrm{f}}=0.55$ at $T=180 \mathrm{~K}$ ). For comparison purposes, the excitation spectra were normalized to the absorption band of the lowest excited singlet state [11]. In all cases, concentrations were low enough in order to exclude intermolecular energy transfer. All experiments reported are independent of concentration.

\section{Molecules without spacer}

The characteristic features for the class of molecules described in this work are displayed by compound 1 . The absorption spectrum of 1 is given in fig. 2 in comparison with those of molecules 2 and 3 representing the donor and acceptor model chromophores.

The three subunits of the supermolecule can be identified. The strong absorption band between 39000 and $41000 \mathrm{~cm}^{-1}$ is characteristic for the $\mathrm{S}_{0} \rightarrow \mathrm{S}_{3}$ absorption of the anthryl group. In the long wavelength region the bands at $24000 \mathrm{~cm}^{-1}$ (B) and

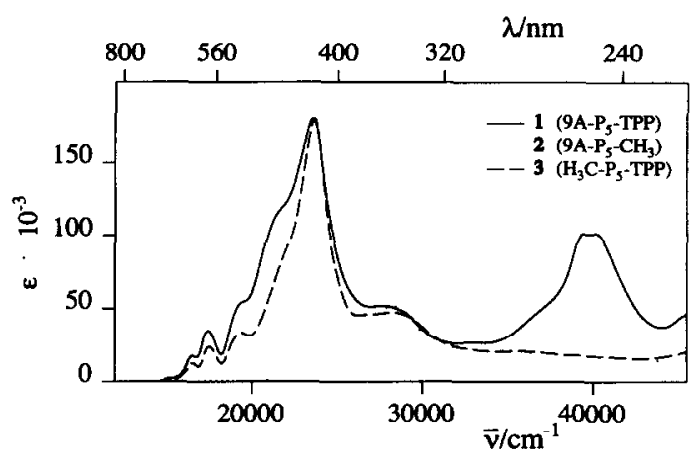

Fig. 2. UV-Vis absorption spectrum of molecule 1 in comparison with those of molecules 2 and 3 representing the right and left part of 1 , respectively. $\epsilon$ given in units of $\mathrm{dm}^{3} \mathrm{~mol}^{-1} \mathrm{~cm}^{-1}$. between 15000 and $20000 \mathrm{~cm}^{-1}$ (Q) are typical for TPP. Superimposed is the polyene absorption. The spectral signature of the polyene contribution to the overall absorption is not as distinct as that of anthracene and TPP. There is a shift to higher energies with decreasing chain length of this part of the absorption spectrum.

It has been shown in detail [13] that particular features of the absorption spectra are due to the formation of the supermolecule, whereas the main absorption characteristics of the individual chromophores remain. So a selective excitation of parts of the molecule is still possible.

The fluorescence spectra of all molecules containing the TPP group are almost identical and obviously characteristic for TPP, see fig. 3 for 1 and 3. Depending on the chain length, the fluorescence quantum yields vary slightly. They decrease from $\eta_{\mathrm{f}}=0.035$ for the $\mathrm{P}_{3}$ compound 7 to 0.015 for 1 (independent of $\left.\lambda_{\text {exc }}\right)$. Independent of the excitation energy, even when exciting the $S_{3}$ transition of $A$, only one fluorescence component (TPP type) is observed. This rcsult is most important and can be interpreted as a consequence of an energy transfer within the supermolecule. Further evidence is given by the excitation spectra in fig. 4. For compound $7\left(9 \mathrm{~A}-\mathrm{P}_{3}-\mathrm{TPP}\right)$ the absorption and excitation spectra for the fluorescence are identical. For molecule 1 (9A-P 5 -TPP), there is a difference in the spectral range between 18000 and $26000 \mathrm{~cm}^{-1}$ indicating a reduced energy transfer to the TPP acceptor if light is absorbed directly by the polyene subunit.

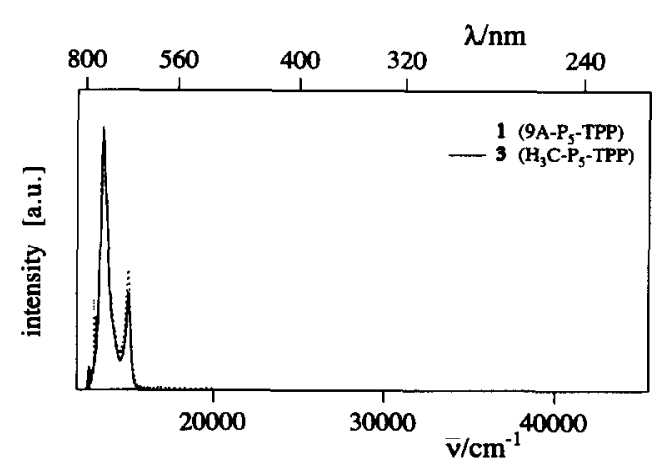

Fig. 3. Fluorescence spectra of compounds 1 and 3 upon excitation with $\lambda=257 \mathrm{~nm}$, normalized to the same maximum intensity given in arbitrary units. 


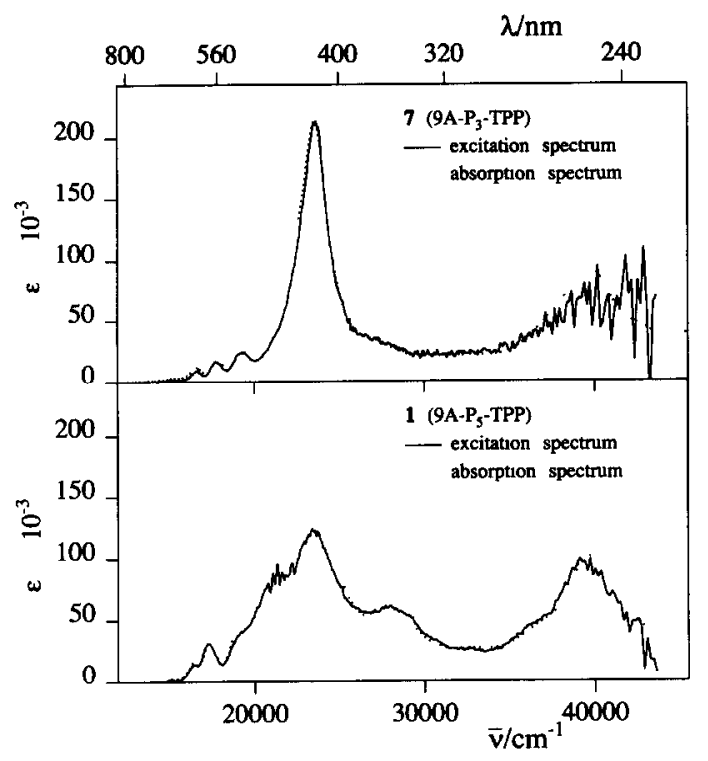

Fig. 4. UV-Vis absorption and excitation spectra of 7 and 1 . The excitation spectra give the fluorescence intensity (in arbitrary units) at $730 \mathrm{~nm}$ as a function of the excitation wavelength; they are normalized to the corresponding absorption spectrum in the spectral region of the TPP Q bands (around $600 \mathrm{~nm}$ ).

\section{Molecules with spacer}

If the intramolecular transfer of energy occurs via the polyene chain, it should be possible to interrupt this process by incorporation of a spacer with electronic energy levels which do not interfere with those important for the intramolecular energy transfer but are located at energies higher than those used for excitation. This is true for the spacer androstane. Examples for the molecules studied are 4, 5 and 6 .

In all these cases the absorption spectra are to a very good approximation superpositions of the individual components of the supermolecule, see fig. 5 for compound 5. Obviously, the spacer-induced separation of their electronic states is almost perfect.

The fluorescence spectra of the molecules with spacer are comparable to those without spacer: whenever TPP is part of the supermolecule, TPP fluorescence, as shown in fig. 3 , is observed. Therefore the same conclusion as in section 4 about an energy transfer can be drawn.

Further evidence is given by examining the excitation spectra in comparison with absorption spectra.

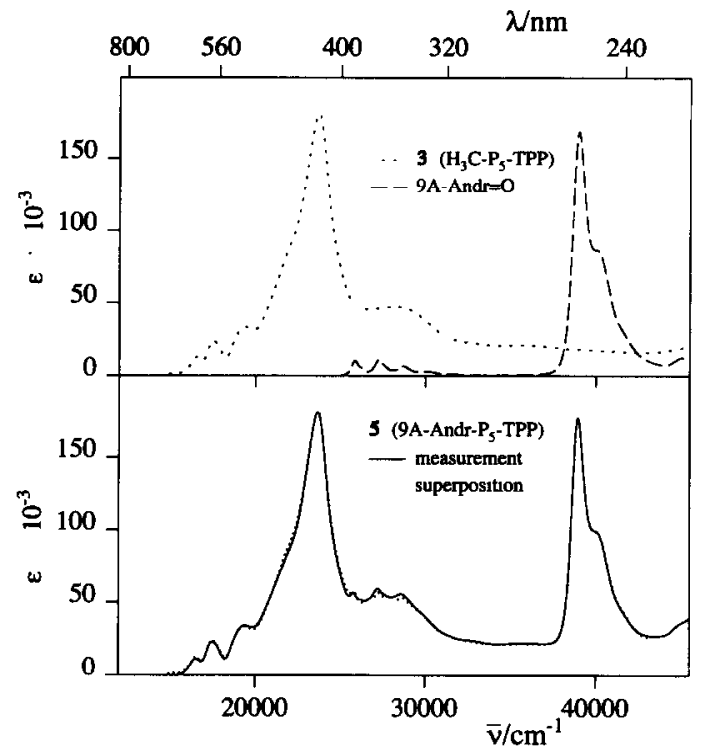

Fig. 5. UV-Vis absorption spectra of 3 and $9 A-A n d r=0$ as well as a comparison of an additive superposition of these spectra with the absorption of 5 .

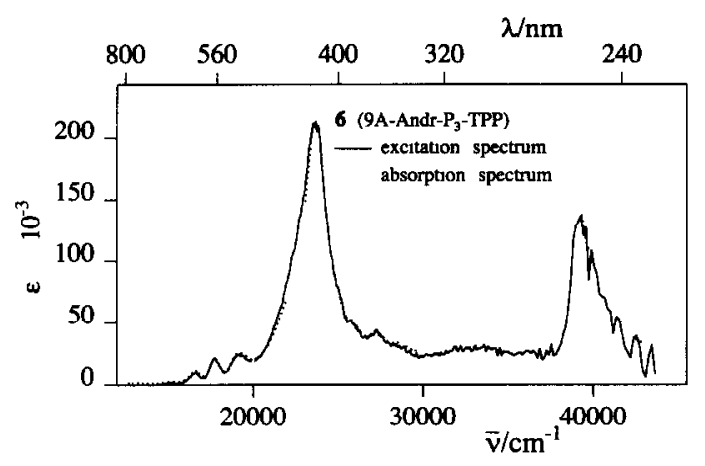

Fig. 6. UV-Vis absorption and excitation spectrum of 6. The excitation spectrum gives the fluorescence intensity (in arbitrary units) at $730 \mathrm{~nm}$ as a function of the excitation wavelength. It is normalized in the same way as the spectra in fig. 4.

For molecule 6 (see fig. 6), the excitation spectrum closely resembles the absorption, with just a slightly reduced relative absorption in the polyene and anthryl $S_{3}$ region. It should be emphasized that with the spacer there is an efficient energy transfer from the A to the TPP subunit. Also the absorption between 30000 and $38000 \mathrm{~cm}^{-1}$ which is mainly due to polyene results in TPP emission.

The excitation spectrum of 5 (fig. 7) is also very 


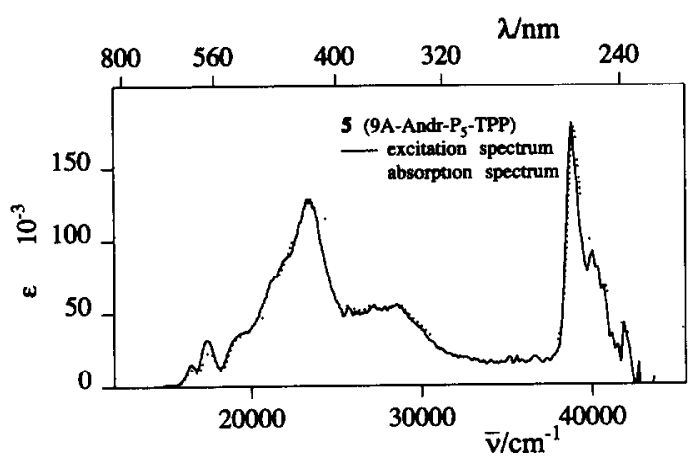

Fig. 7. UV-Vis absorption and excitation spectrum of 5. The excitation spectrum gives the fluorescence intensity (in arbitrary units) at $730 \mathrm{~nm}$ as a function of the excitation wavelength. It is normalized in the same way as the spectra in fig. 4 .

similar to its absorption spectrum. In this case, there is a slight difference around the B-band of TPP at about $24000 \mathrm{~cm}^{-1}$. This can be interpreted as a reduced transfer upon the polyene absorption which is red-shifted at this chain length (see also fig. 4) as compared to 6 . This reduction is probably due to a competing radiationless relaxation of the polyene chain when excited directly. It is well known that polyene systems generally undergo isomerisation about the double bond in the excited state and this could be a reason for a low quantum yield.

\section{Discussion: The mechanism of energy transfer}

From the measurements presented in the previous sections it is clear that in all molecules terminally substituted with A and TPP there is an efficient energy transfer from A to TPP when the exciting light is absorbed by the anthryl group. Also upon direct excitation of the polyene energy transfer to TPP is detected. Intermolecular transfer cannot be responsible for these observations since the transfer efficiencies are independent on the concentration. Therefore the energy transfer must be due to an intramolecular mechanism.

One possible mechanism is fast intramolecular relaxation (internal conversion) within the excited states of the subunits down to the lowest excited singlet state of the supermolecule. Because the absorbing (A) and the emitting (TPP) subunits are spa- tially separated, an unidirectional intramolecular energy transfer using the electronic levels of all three subunits results. A special through-bond transfer mechanism using a specific electronic level of the polyene chain cannot be identified.

Additional information comes from the molecules with spacer. Obviously the spacer interrupts the overlapping excited states of the other partners of the supermolecule. An intramolecular relaxation between the two ends of the supermolecule across the spacer could be mediated only by vibronic coupling. But due to the fact that the electronic excited levels of the spacer are much higher than those of the other subunits concerned with intramolecular relaxation, this process should not be very efficient.

An alternative electronic mechanism making an energy transfer across the spacer possible is dipoledipole interaction also known as Förster transfer [14]. Precise values for the Förster radius, this is the critical distance for energy transfer, cannot be calculated with accuracy since neither the overlap of donor emission and acceptor absorption nor the donor fluorescence quantum yield in the supermolecule which are needed for that calculation are known precisely enough. With plausible assumptions, Förster radii between 60 and $75 \AA$ are calculated [8]. These values are much larger than the donor-acceptor distance of about $35 \AA$ even in the largest supermolecules investigated in this work. So generalized Förster transfer seems to be the mechanism by which the observed unidirectional flow of energy within the supermolecules can be understood.

\section{Conclusion}

In conclusion, terminally donor-acceptor substituted polyenes do not represent the best candidates for "molecular wires" designed to transfer excitation energy in a given direction. Though the transfer efficiency is high, competing radiationless processes are quite important. In the present systems this is due to the low fluorescence quantum yield of the acceptor TPP in addition to radiationless deactivation of the polyene subunits. The most plausible intramolecular energy transfer mechanism other than intramolecular relaxation is Förster-type transfer. In order to clarify this further, measurements in frozen matrix at 
lower temperatures are under way. Modifications of the supermolecules could lead to systems more suitable for unidirectional energy transfer in donorspacer-acceptor supermolecules.

\section{Acknowledgement}

This work was supported by the Deutsche Forschungsgemeinschaft, SFB 329, and by the Fonds der Chemischen Industrie.

\section{References}

[1] F. Effenberger and H.C. Wolf, New J. Chem. 15 (1991) 117. [2] H.C. Wolf, in: Molecular electronics - science and technology, ed. A. Aviram (AIP, New York, 1992) p. 237.

[3] M.R. Wasielewski, D.G. Johnson, W.A. Svec, K.M. Kersey, D.E. Cragg and D.W. Minsek, in: Photochemical energy conversion, eds. J.R. Norris and D. Meisel (Elsevier, Amsterdam, 1988) p. 135;
A. Slama-Schwok, M. Blanchard-Desce and J.M. Lehn, J. Phys. Chem. 94 (1990) 3894.

[4] S. Maier, H. Port, H.C. Wolf, F. Effenberger and H. Schlosser, Synth. Metals 29 (1989) E 517.

[5] F. Effenberger, H. Schlosser, P. Bäuerle, St. Maier, H. Port and H.C. Wolf, Angew. Chem. 100 (1988) 274.

[6] G. Blessing, N. Holl, H. Port, H.C. Wolf, F. Effenberger, T. Kesmarszky and H. Schlosser, Mol. Cryst. Liq. Cryst. 183 (1990) 21.

[7] N. Holl, P. Emele, H. Port, H.C. Wolf, H. Strobel, T. Kesmarszky and F. Effenberger, Chem. Phys. Letters 205 (1993) 25.

[8] H. Strobel, Dissertation, Universität Stuttgart (1992).

[9] F. Effenberger and H. Schlosser, Synthesis 1990, 1085;

F. Effenberger and T. Kesmarszky, Chem. Ber. 125 (1992) 2103;

M. Koch, Forschungspraktikum Universität Stuttgart (1989).

[10] F. Effenberger and H. Strobel, Chem. Ber., in press.

[11] N. Holl, Dissertation, Unıversität Stuttgart (1992).

[12] S. Ates and A. Yildiz, J. Chem. Soc. Faraday Trans I 79 (1983) 2853.

[13] P. Gribi, G. Isenmann, E. Sigmund, G. Quapil, N. Holl and H. Port, J. Chem. Phys. 98 (1993) 7969.

[14] T. Förster, Z. Naturforsch. 4a (1949) 321. 